$63^{\text {ème }}$ Congrès de la SFCO, 03003 (2015)

DOI:10.1051/sfco/20156303003

(C) Owned by the authors, published by EDP Sciences, 2015

\title{
Évaluation de l'angiogenèse tumorale dans les carcinomes épidermoïdes de la cavité buccale : étude immunohistochimique
}

\author{
Bouguezzi A***, Sriha B****, Selmi $\mathrm{J}^{* * * *}$, Ben Amor $\mathrm{F}^{* * * * * *}$ \\ * Service de médecine et chirurgie buccales, Clinique dentaire universitaire, Monastir, Tunisie \\ ** Laboratoire de Recherche Santé Orale et Réhabilitation Bucco-Faciale (LR12ES11), FMDM-Université, \\ Monastir, Tunisie \\ *** Service des consultations externes, Clinique dentaire universitaire, Monastir, Tunisie \\ **** Service de cytologie et anatomie pathologiques, Hôpital universitaire Farhat Hached, Sousse, Tunisie
}

Le cancer des voies aéro digestives supérieurs (VADS) est difficile à traiter en raison de sa localisation et de sa forte tendance à envahir les tissus environnants et à se disséminer dans les nodules lymphatiques. De plus, l'évolution de la maladie est difficile à prédire avec les outils conventionnels (clinique, histopathologie), c'est pourquoi la recherche de biomarqueur est nécessaire pour prédire l'évolution de la maladie mais également pour faire profiter les patients d'une thérapie plus adaptée. L'objectif de ce travail est de comparer la densité vasculaire péri-tumorale dans une série de carcinomes épidermoïdes de la cavité buccale en fonction du sexe, âge des patients, localisation tumorale, stade, métastases ganglionnaires, degré de différenciation, à l'aide de deux anticorps anti CD31 et anti CD34, et de déterminer sa signification pronostique. La densité vasculaire péri-tumorale est mesurée par deux observateurs indépendants, par comptage de toutes les structures CD34+ et CD $31+$, le résultat est exprimé par le nombre moyen de structures CD34+ et CD31+/mm2. L'étude porte sur 50 cas de carcinomes épidermoïdes de la cavité buccale, dont 21 non métastatiques (N-) et 29 avec des métastases ganglionnaires $(\mathrm{N}+)$. La répartition en fonction de la topographie était la suivante : gingivo mandibulaire $(n=13)$, gingivo maxillaire $(n=3)$, lèvre $(n=20)$, langue $(n=9)$, joue interne $(n=4)$, plancher buccal $(\mathrm{n}=1)$. Nous avons montré que le marquage des vaisseaux péri tumoraux des carcinomes épidermoïdes de la cavité orale est très variable selon l'anticorps utilisé, il est plus important pour l'anticorps anti CD34 que pour l'anticorps anti CD31 (la moyenne de la DMV pour l'anticorps anti CD34 est de $22.75 \pm 19.7$, tandis que pour l'anticorps anti CD31 est de 13,74 \pm 28.92). On trouve une corrélation statistiquement significative entre l'âge des patients et la densité vasculaire péritumorale et ceci avec l'anticorps anti CD34 ( $\mathrm{p}=0.027)$. La densité vasculaire est plus importante dans la périphérie des tumeurs avec envahissement ganglionnaire que dans les tumeurs non métastatiques et ceci pour les deux anticorps anti CD31 et anti CD34, cette relation n'a pas été corrélée statistiquement (p respectivement 0.13 et 0.062). Malgré l'hétérogénéité et la complexité des mécanismes en jeu, la quantification est aujourd'hui un facteur pronostique indépendant de la plupart des tumeurs : plus celles-ci sont vascularisées, plus le risque de dissémination métastatique augmente.

Nom et adresse du conférencier

Adel BOUGUEZZI

Service de médecine et chirurgie buccales, Clinique dentaire universitaire Monastir (Tunisie)

dr-adel@live.fr

This is an Open Access article distributed under the terms of the Creative Commons Attribution License 4.0, which permits unrestricted use, distribution, and reproduction in any medium, provided the original work is properly cited. 